\title{
INTERACTIONS BETWEEN ACTIVE ELEMENTS (OBSERVATIONS ON LILLIE'S NERVE MODEL)
}

\author{
By KAZUMI YAMAGIWA \\ Tokyo Medico-Dental College, Tokyo
}

\section{INTRODUCTION}

In the previous work on Lillie's nerve model, we found that the law of isolated conduction holds very good in the model (Yamagiwa ${ }^{1)}$ ), that is, the cores appear as if they were independent from and indifferent to each other as far as the activation conduction is concerned. In fact, however, they cannot be quite so, because they are not isolated electrically and the activation current emanating from an activated core flows really into the others. The point is that the current is of such a small intensity that it cannot activate them in the usual conditions. The object of the present work is to examine the effects of this weak electric current penetrating the neighbouring cores upon their activation conductions. The true object lies of course in the hope that it may help us more or less in the studies on, or in the understanding of, nervous conduction, where the electric interaction among the fibres must be playing a role as in the model.

As to the influences of active fibres upon the neighbouring resting ones, we have had rather few informations. If we exclude those on the non-isolated conduction, which were quoted in the former report (Yamagiwa ${ }^{1)}$ ), we have perhaps only those of Otani ${ }^{2)}$, Katz and Schmitt ${ }^{3)}$, Blair and Erlanger ${ }^{4}$, Renshaw and Therman ${ }^{5)}$, and Marrazzi and Lorente de No. ${ }^{6}$ They are all concerned with the change of excitability of the resting fibres, except that of Katz and Schmitt; which dealt also with the effect upon the conduction velocity. They discovered the phenomenon of "synchronization" of the impulses in two adjacent nerve fibres of slightly different conduction velocities, and also the phenomenon of "slowing" of the impulses, when they are advancing entirely "in step". The present work, which was performed independently during the war time without being aware of their work, resulted partly in reconfirming their important findings and also in revealing some other facts, which will be described briefly in the following pages.

\section{Methods}

A long columnal groove, ca $47 \mathrm{~cm}, 2.5 \mathrm{~cm}$ and $1 \mathrm{~cm}$ in length, breadth and depth respectively, was made in a large block of paraffin, and it was filled with strong nitric acid for two or more cores to be laid in parallel within it. One end of each core was bent in a proper shape to be led to small Hg-pots, which are separated from one another and from the acid by paraffin walls (Fig. 1).

In the first group of observations, several combinations of cores were chosen at random; for example, cores of different thicknesses, cores of different immersion times or cores made from different samples, etc., in order to have cores of different conduction velocities. In the second group, cores similarly made from one and the same sample , 
were employed on account of their having nearly the same conduction velocities. In either case, the stimulus was given at one end of the core, as illustrated in the figure, by means of a Zinc metal touching. If we want to stimulate the cores separately, we keep the keys $K_{1}$ and $K_{2}$ open, and when a simultaneous stimulation is wanted, the keys are kept closed.

\section{The first group of observations (The case when the cores are connected metalically at one end).}

If the cores are brought into metalic connection at one end, they are in the situation to give rise to the "apparent non-isolated conduction" described in the former report (Yamagiwa ${ }^{1)}$ ). They did so really when the conditions were fulfilled, and this is nothing but the extreme case of interaction manifested. There is, however, another thing to pay attention to, that is, the effect of the weak electrlc current, which is not strong enough to activate the neighbours. It is our object here to examine this, and we planned to do so by making the distances between the cores properly large or by employing cores of relatively short immersion times.

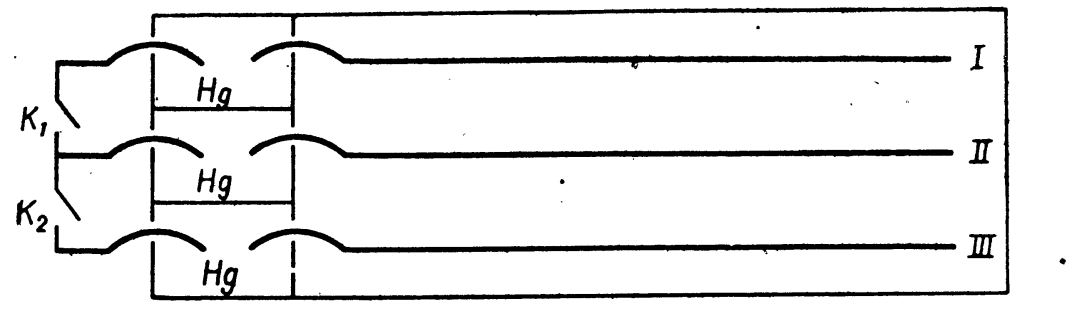

Fig. 1

A) The conduction in cores of different conduction velocities.

Two or three cores chosen at random were laid in parallel in a bath, properly apart from one another. It was ascertained beforehand that the cores commit certainly the isolated conduction. Now, after a certain immersion time, $t$, the conduction time of each core was measured by stimulating them one by one separately. Then, after another $t$, all the cores were stimulated simultaneously by applying a Zinc-stimulus to one of the cores, with the keys $\mathrm{K}_{1}$ and $\mathrm{K}_{2}$ closed, and thus the conductions of all the waves starting together were brought under examination. The result was, quite unexpectedly, that all the waves were conducted with one and the same velocity, in spite of the deviating values of the individual ones. The experiments were repeated many times with several combinations of the cores, but the results were always the same. Instead of giving the rather complicated protocols as they are, it will be better to give the points of the results obtained in the simple case of two cores. They are as follows:

1) If two cores of a "large" and a "small" conduction velocities are connected metalically at one end and stimulated simultaneously, the activation waves are conducted with a certain "common" velocity, which is smaller than the "large" one and larger than the "small" one. 
2) In the above case, the distance travelled in the core with the "small" velocity gets more or less larger than that in the case of single activation. This phenomenon takes place when the velocity is so small in that core that the wave is transmitted with decrement when activated alone.

We can express the results also in the following terms: the quick core gives some velocity and distance to the slow one, at the cost of its own velocity. And we see, as a natural sequence from that, that generally in a group of cores with a metalic connection at one end, the activation waves are conducted with a certain "mean" velocity when they are activated simultaneously, or, in other words, that the several velocities

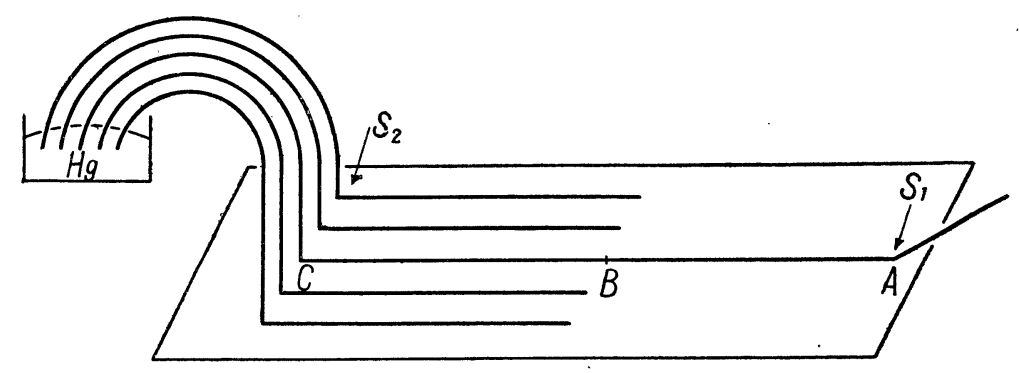

Fig. 2

of each individual cores are levelled to an average one. Here we have a sort of synchronization with regard to activation conduction, a remarkable thing to be noticed indeed.

B) The conduction in cores of one and the same conduction velocity.

When we were sure of the levelling of the velocities, we wondered at once, what will happen, then, if the cores were of the same conduction velocity from the beginning. To examine this, cores were made freshly from one and the same sample, taking every care in the way of polishing and other treatments to make them as similar as possible in their velocities and other properties.

The first examinations were made with two cores, and we found again the waves being conducted with a certain common velocity as before. But it mas beyond our expectation that this "common" velocity was definitely a little smaller than the individual ones. In order to reexamine this and, at the same time, to examine whether the phenomenon has anything to do with the number of the cores, the cores were arranged in such a way as illustrated in Fig 2.

Fig 2 represents five cores laid in parallel, of which one is long $(40 \mathrm{~cm})$ and four are half as long $(20 \mathrm{~cm})$. The distances between two neighbouring cores were $2-3 \mathrm{~mm}$, a distance short enough to give to the "apparent non-isolated" conduction (Yamagiwa). Now, a stimulus is applied to the right end, $A$, of the long core $\left(S_{1}\right.$ in the figure). At the moment of arrival of the wave at the middle point, $\mathrm{B}$, we see all the short cores getting into activation simultaneously, as the result of the apparent non-isolated conduction, and thereafter we have five waves travelling in step towards the left end, C. It was the object here, to measure the conduction times for the region $A B$ as well as for 
the region $\mathrm{BC}$, to compare them with each other. The measurements were done by means of two stop-watches held in both hands. An example of the series of data obtained is given in Table 1 with its graphical illustration in Fig. 3.

Table 1

\begin{tabular}{|c|c|c|c|c|c|c|c|}
\hline \multirow[t]{2}{*}{$\begin{array}{l}\text { Number of } \\
\text { short cores }\end{array}$} & \multirow[t]{2}{*}{$\mathrm{t}$} & \multirow[t]{2}{*}{ Stimulus } & \multicolumn{2}{|c|}{$\begin{array}{l}\text { Cond. time } \\
\left.\text { (in } 1^{\prime \prime} / 5\right) \text { for }\end{array}$} & \multirow[t]{2}{*}{$\mathrm{BC} / \mathrm{AB}$} & \multirow[t]{2}{*}{ Mean } & \multirow[t]{2}{*}{ Remarks } \\
\hline & & & $\mathrm{AB}$ & $\mathrm{BC}$ & & & \\
\hline 9 & $\begin{array}{c}2^{\mathrm{h}} 5^{\prime 1} \\
10^{\prime} \\
15^{\prime} \\
20^{\prime}\end{array}$ & $\mathrm{S}_{1}$ & $\begin{array}{l}- \\
3 \\
3 \\
3\end{array}$ & $\begin{array}{l}\overline{11} \\
11 \\
12\end{array}$ & $\begin{array}{l}- \\
3.7 \\
3.7 \\
4.0\end{array}$ & 3.8 & $\begin{array}{l}\text { A) conditioning } \\
\text { stimulus }\end{array}$ \\
\hline 7 & $\begin{array}{l}\left.35^{\prime 2}\right) \\
40^{\prime} \\
45^{\prime} \\
50^{\prime}\end{array}$ & $\mathrm{S}_{\curlywedge}$ & $\begin{array}{l}- \\
4 \\
5 \\
4\end{array}$ & $\begin{array}{l}- \\
14 \\
15 \\
16\end{array}$ & $\begin{array}{l}- \\
3.5 \\
3.0 \\
4.0\end{array}$ & 3.5 & $\begin{array}{l}\text { A conditioning } \\
\text { stimulus }\end{array}$ \\
\hline 5 & $\begin{array}{r}55^{\prime} \\
60^{\prime} \\
3 \mathrm{~h} \quad 5^{\prime}\end{array}$ & S. & $\begin{array}{l}5 \\
5 \\
5\end{array}$ & $\begin{array}{l}16 \\
16 \\
18\end{array}$ & $\begin{array}{l}3.2 \\
3.2 \\
3.6\end{array}$ & 3.3 & \\
\hline 3 & $\begin{array}{l}10^{\prime} \\
15^{\prime} \\
20^{\prime}\end{array}$ & $\mathbf{S}$ & $\begin{array}{l}5 \\
5 \\
5\end{array}$ & $\begin{array}{r}9 \\
12 \\
11\end{array}$ & $\begin{array}{l}1.8 \\
2.4 \\
2.2\end{array}$ & 2.1 & \\
\hline 2 & $\begin{array}{l}25^{\prime} \\
30^{\prime} \\
35^{\prime}\end{array}$ & $\mathrm{S}_{1}$ & $\begin{array}{l}6 \\
7 \\
6\end{array}$ & $\begin{array}{l}9 \\
9 \\
9\end{array}$ & $\begin{array}{l}1.5 \\
1.3 \\
1.5\end{array}$ & 1.4 & \\
\hline 03) & $\begin{array}{l}40^{\prime} \\
45^{\prime} \\
50^{\prime}\end{array}$ & $\mathrm{S}_{1}$ & $\begin{array}{l}7 \\
7 \\
7\end{array}$ & $\begin{array}{l}7 \\
7 \\
7\end{array}$ & $\begin{array}{l}1.0 \\
1.0 \\
1.0\end{array}$ & 1.0 & $\begin{array}{l}\text { O) } \\
\text { corly the long } \\
\end{array}$ \\
\hline
\end{tabular}

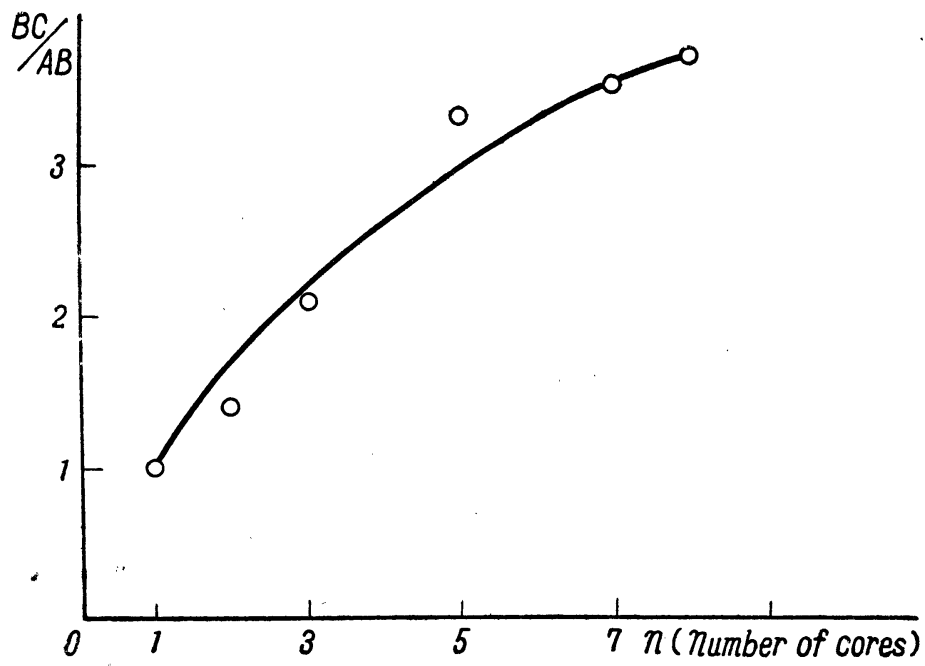

Fig. 3 
The data obtained show us clearly that the larger the number of the cores is, the larger is the value of the ratio $\mathrm{BC} / \mathrm{AB}$, the conduction time for the region $\mathrm{BC}$ against that in the region $\mathrm{AB}$. That is to say, a larger number of the cores has a larger effect in making the velocity decrease.

The stimulus was often given to the left end of the core for trial $\left(S_{2}\right.$ in Fig. 2 ), but there was no change in the result.

\section{The second group of observations (The case when the cores are disconnected)}

It was confirmed in the above expriment, that the conduction velocity in a multiple core is smaller than that in the single core (Refer to Fig. 2). There are two factors now, which might be the causes of the phenomenon, namely, 1) the co-existence of other cores, and 2) their simultaneous activations. Are they both responsible or just one of them?

What we have to do first of all for answering this question, is to repeat the experiments represented by Fig. 2 with the cores disconnected, because then the short cores change into those simply co-existing beside the long core. A series of new data thus obtained is given in Table 2 as an example. In the experiments I-IV in Table 2, the

Table 2

\begin{tabular}{|c|c|c|c|c|}
\hline Experiments & $\begin{array}{l}\text { Number of } \\
\text { short cores }\end{array}$ & $\mathrm{BC} / \mathrm{AB}$ & Mean & Remarks . \\
\hline I & 0 & $\begin{array}{l}1.0 \\
0.8 \\
0.8\end{array}$ & $0 . \dot{9}$ & $\begin{array}{l}\text { Only the long } \\
\text { core existing }\end{array}$ \\
\hline II & 2 & $\begin{array}{l}0.8 \\
1.2 \\
1.2\end{array}$ & 1.1 & $\begin{array}{l}\text { The isolated } \\
\text { conduction taking place }\end{array}$ \\
\hline III & 4 & $\begin{array}{l}1.2 \\
0.8 \\
1.0\end{array}$ & 1.0 & " \\
\hline IV & 6 & $\begin{array}{l}1.2 \\
1.2 \\
0.8\end{array}$ & 1.1 & 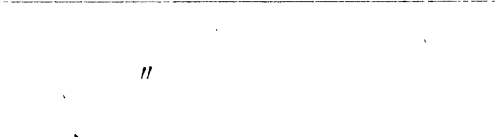 \\
\hline $\mathrm{V}$ & 6 & $\begin{array}{l}2.6 \\
2.6 \\
2.0\end{array}$ & ${ }^{2.2}$ & $\begin{array}{l}\text { All the cores connected. } \\
\text { Simultaneous activations taking } \\
\text { place in all the cores. }\end{array}$ \\
\hline VI & 6 & $\begin{array}{l}1.6 \\
1.8 \\
1.6\end{array}$ & 1.7 & $\begin{array}{l}\text { All the short cores connected except } \\
\text { the long one. The stimulus was } \\
\text { given to the long one and to the } \\
\text { adjacent short one at the left end } \\
\mathrm{C} \text {, by which all the cores were } \\
\text { activated simultaneously. }\end{array}$ \\
\hline
\end{tabular}


ratio $\mathrm{BC} / \mathrm{AB}$ is nearly unity as a whole, which means that the simple co-existence of resting cores exerts no influence upon the conduction velocity in an activated core. In the experiment $\mathrm{V}$, on the other hand, where all the seven cores are connected metalically, the ratio attains as large as 2.2. In the experiment VI, the connection was made among the short cores only, leaving the long one alone. The stimulus was applied to the long core and to the adjacent short core simultaneouly at the left end, $\mathrm{C}$ (Fig. 2) by means of a relatively large Zinc foil. So the short cores as a whole stand here as a simple neighbour to the long core, but not as a quiet neighbour because it gets activated together with the long core. The ratio obtained in this case was 1.7 , a value distinctly larger than those in the experiments I-IV, although it is smaller than that in the experiment V. (The difference between 2.2 and 1.7 might have been one due to the presence and absence of connection between the long and short cores).

We learnt thus 1) that the simple co-existence of cores is indifferent to the conduction velocity in another core activated, and 2) that the smaller velocity in the multiple core is due to the simultaneous activations of the partner cores, but not due to their co-existence nor to the metalic connection with the long core.

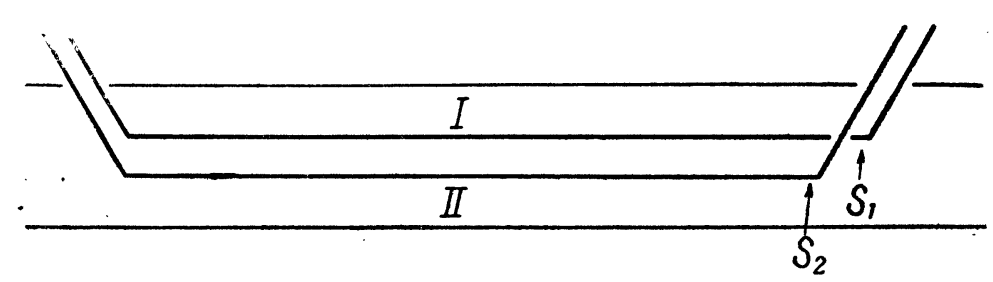

Fig. 4

\section{The third group of observations (Precise reexaminations with two cores)}

The above observations taught us clearly that the cores laid in parallel in short distances do interact intimately, although they appear to be quite indifferent to each other, committing the isolated conduction usually. It is indeed a fact full of suggestions for us as to the nervous conduction and related physiological phenomena, and on that very account, we ought to be quite careful in accepting it. It was undertaken, therefore, to reexamine the things precisely under some simple conditions. We limited the number of cores to two, on which we repeated carefully the same kind of experiments which we had performed before. And as the result, we came to the same conclusions as before, and in addition, we found a new fact of interaction which will be worth while to be noted here.

1. Methods

Two cores of an equal length $(40 \mathrm{~cm})$ were made from one and the same sample. Cares were taken in the process of preparation to make the conduction velocities as near as possible to each other. It was yet impossible to have them perfectly equal, and moreover they changed their values gradually with time, and that with different grades in different cores. It was disturbing indeed but sometimes it turned out to be very 
useful, too. (Refer to Figg. 5 and 6 .

The cores thus prepared were laid in parallel in a bath, ca. $5 \mathrm{~mm}$ apart. Both ends were all bent upwards to be held in the air unconnected (Fig. 4).

By applying the stimuli $S_{1}$ and $S_{2}$ one after another, the conduction times for the whole lengths of each core were first measured at a certain immersion time. Then after another immersion time of the same duration, the both cores were activated simultaneously by means of a Zinc foil, which was wide enough to mount them. The waves

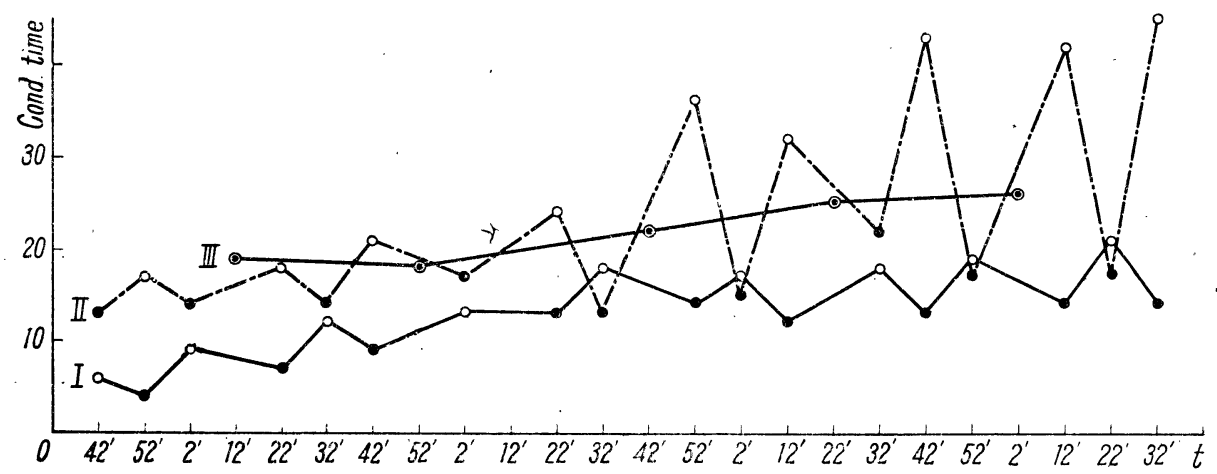

Fig. 5

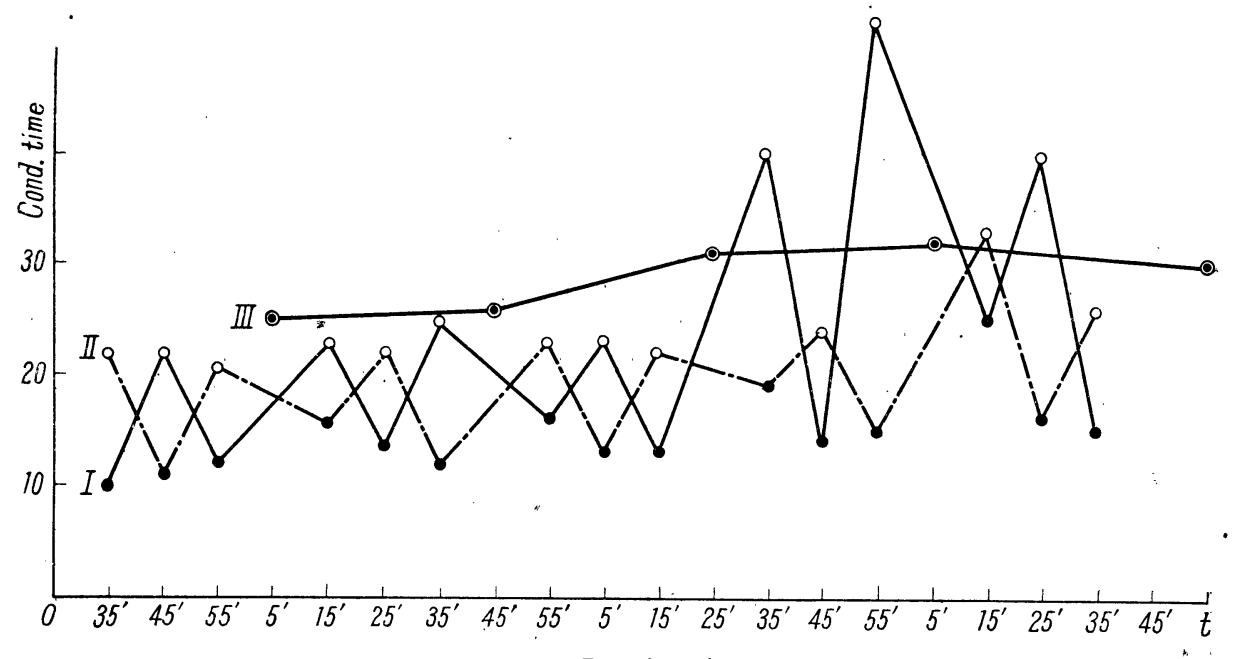

Fig. 6

evoked were seen to proceed certainly with one and the same velocity, as was observed before. This "common" velocity was measured out to be compared with the individual ones.

2) The results

In spite of the simplicity of the method, the data obtained, particularly those for the individual velocties, were rather irregular and indefinite to begin with. After several kinds of trial experiments and after much patience, it was noticed occasionally that the 
conduction time of a core gets definitely smaller (that is, the conduction velocity gets larger), if it is activated directly after the activation of the neighbouring core. Till then, we were giving the stimuli $S_{1}$ and $S_{2}$ successively with a very short interval ( $2 \cdot 3$ seconds) to finish up the measurements as quickly as possible. The first stimulus given was thereby sometimes $S_{1}$ and sometimes $S_{2}$. This was the cause of the confusion of the results. It disturbed us very much, but it resulted in making us find a sort of interaction in the disturbance itself. Taking the finding into consideration, we settled the way of stimulation properly and we could now get regular and definite results, of which two examples are given in Fig. 5 and Fig. 6.

In the figures, the curves I and II represent the conduction times of the cores I and II respectively, which were measured at every ten minutes throughout a few hours. Every time a pair of measurements were done on two cores successively, whereby the core I or II was chosen alternately as the first one to be stimulated. The marks $\circ$ and - are for denoting the conduction times of each core thus measured before and after the other core.

As seen in the figures, both curves run regularly in zig-zag, showing that the conduction time of a core gets always a little shortened if measured directly $(2-3 \mathrm{sec}$. $)$ after

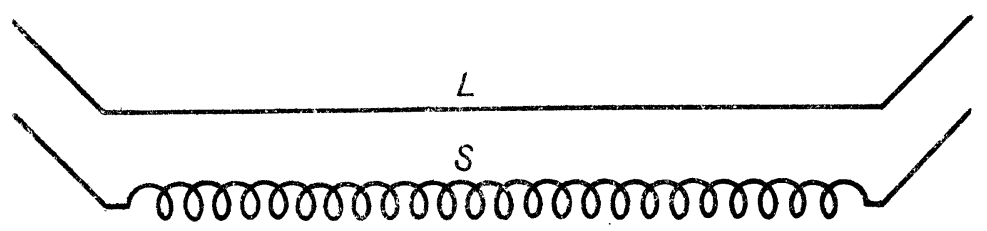

Fig. 7

the activation of the other. Next, the curve III in the figures represents the "common" conduction time, with which the waves are transmitted in both cores when activated simultaneously. It was measured at every 30 or 40 minutes. We see the curve III running above the curves $I$ and II in the former haif, and running between them in the latter half. These facts are not new to us, because we were already acquainted with the former in our experiments on cores of the same velocities, and with the latter in the experiments on cores of different velocities. The conditions in the present experimpnts nicely correspond to those of old ones, because the individual conduction velocities werè very near to each other to begin with, and happened to be much different later, although the reason is not clear. Anyway, the curve III tells us again the same things which were told to us before.

We wish to add a special case observed, in which a linear and a spring-shàped core were laid in parallel (Fig. 7). The apparent lengths of the cores $L$ and $S$ in the figure were both ca. $45 \mathrm{~cm}$, but actually $\mathrm{S}$ was 2-6 times as long as $\mathrm{L}$. They. were prepared from one and the same sample and placed in a bath ca. $1.5 \mathrm{~cm}$ apart. Now, the conduction time of S, measured separately; was found very much larger than that of L, as it was expected from the beginning (It was, however, much smaller than propor- 
tional to the actual length. Details will be given in a later paper.). But when. the cores were stimulated simultaneously, the waves were found again to proceed with a "common" velocity of a certain intermediate value.

\section{Considerations}

We will first summarise the results obtained:

1) If cores of different conduction velocities are stimulated simultaneously $y_{\bullet}$ the activation waves are all comducted with a certain mean velocity, which is smaller than the largest and larger than the smallest one.

2) If the individual velocities are the same or nearly the same in the above case, the cores transmit the waves with a "common" velocity, which is smaller than either of the individual ones. The decrease of the velocity is thereby the greater, the larger the number of the cores is.

3) If a core is stimulated directly (practically 2-3 sec.) after the activation of a neighbouring core, the activation wave is transmitted with a velcoity a little larger than usual.

4) These facts are independent from whether the cores are connected or disconnected at one end, but they fail to occur when the immersion time is too short or the distance between the cores is too large.

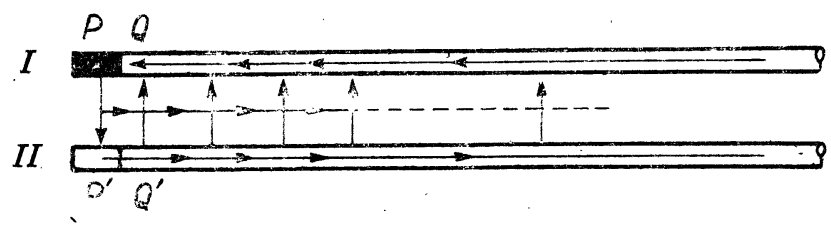

Fig. 8

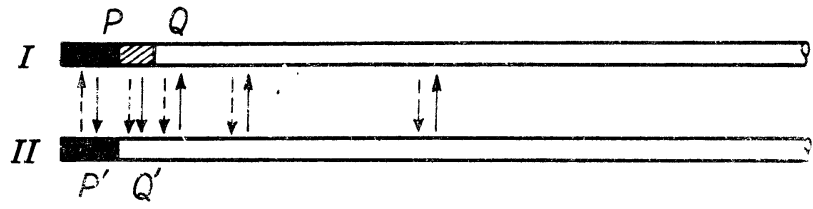

Fig. 9

- The metalic connection between the cores was first considered to be an important factor for the phenomena observed, but it was proved later not to be so. (Perhaps it is an enforcing factor, making the circuit resistance smaller and thus the electric interaction the stronger.) The whole facts should then be accounted for as a matter of pure interaction between the cores standing side by side.

Let us take, for the sake of simplicity, the case of two cores of equal conduction velocities laid in parallel, to begin with (Fig. 8). Suppose that the edge point (exactly to say, some edge region), $\mathrm{P}$, of the core $\mathrm{I}$ is-activated by an external force. The activation current will emanate from $\mathrm{P}$ and flow in such directions as indicated by. the 
arrows in the figure. A fraction of the current enters the core II at $\mathrm{P}^{\prime}$, which is facing $\mathrm{P}$, and leaves it at $\mathrm{Q}^{\prime}$ (and other parts). This current acts on $\mathrm{P}^{\prime}$ activatingly and on $\mathrm{Q}^{\prime}$ (and others) passivatingly. Just the same actions come from the core II on $\mathrm{P}$ and $\mathrm{Q}$ (and others) of the core I, when the core II is activated. Hence, if $\mathrm{P}$ and $\mathrm{P}^{\prime}$ are activated simultaneously, we have as the result some inhibitory effects being exerted on both $\mathrm{Q}$ and $\mathrm{Q}$ '. This will bring forward the "slowing" of both activation waves. We see from this consideration that a core activated alone acts more or less activatingly upon a co-existing core, while two cores activated together act more or less inhibitorily on each other. The result 2) above mentioned can be easily understood from this point of view.

If now, the cores are different in their velocities, the wave front in one core is always to lead that in the other core. Let this tendency be represented by the shadowed part of the active region in the core I (Fig. 9.). In Fig. 9 the arrows in full lines are indicating the flow of the current from the core $I$, and the arrows in broken lines that of from the core II. As it will be seen from the figure, the activation in the region $\mathrm{Q}^{\prime}$ is favoured by the current coming from the shadowed part, which will result in pushing the wave front in the core II a step nearer to that in the core I, while the current from the core II has just the contrary effect on the core I, because it leaves the core I in the region $\mathrm{Q}$ (and following parts) where the current emanating from the region $\mathrm{P}$ is coming in to activate it. This will act to pull back the wave front in it. The levelling of the velocities of two or, generally, more cores will thus be brought forward. (The result 1). above mentioned). Why, however, does the "slowing" occur, instead of a simple levelling, in two cores of slightly different velocities? It is perhaps like this: firstly, the velocities are levelled to an average one, and then secondly, the "slowing" takes place owing to mutual inhibitory interaction. These two processes will happen in two cores of different velocities in general, but if the cores are of greatly different velocities at the beginning, the final velocity can be still larger than the small one, even if reduced. after the levelling.

Finally, the fact 3) will be, we think, due to the residual of the anodic (activating) effect left behind in the resting core by the sweep of an activation wave along a neighbouring core. Precise examinations as to this effect and related things, for example the question whether there are depressing phases before and after the supernormal phase, or not, were not carried out in the present work.

Now, whether the above explanations are right or not, is of little matter. The important point is that the observed facts themselves are very note-worthy and suggestive. Hasn't the result 3), for instance, anything to do with the facilitation and inhibition of reflexes at the simultaneous or successive application of two stimuli on two spots of the skin?' Several phase relations between the two impulses arriving at the final common path, which is composed of several conducting units, from two different routelets, seem to be promising to explain some experienced facts, and the more so, if there are, besides the supernormal phase, depressing phase or phases in course of interaction. Further, if the fact 1 ) is also the case in the nerve, it will be a useful mechanism for 
making the impulses in a group of fibres arrive at their end-organ simultaneously. The conduction volocities of nerve fibres are known to depend on their thicknesses, the temperature, the internal and external conditions, etc. But it seems to be a matter of great improbability that, for example, all the motor fibres supplying a muscle are exactly the same in all these conditions. How, then, can the muscle fibres be brought into action simultaneously at a voluntary contraction? Isn't there the same principle of velocity levelling there as in the model? An objection will certainly arise, among others, that the nerve fibres are insulated from each other and cannot interact so easily as the cores in the model. It is quite right, but the insulation is never strict even in the medullated fibres, and we think there is a large possibility of interaction there. Not only it is possible theoretically, but also it was proved actually by many workers quoted in the introduction. A particularly interesting and important information was brought by Katz and Schmitt ${ }^{3}$ ) (1940), who found the "synchronization", etc., of the impulses in two adjacent nerve fibres, just as we did in the model independently.

If it is supposed, then, the levelling of the velocities is a general happening in the nerve, it seems to contradict Erlanger and Gasser's famous finding that in a nerve trunk, fibres of nearly the same diameter make a group to transmit impulses with a certain velocity of its own. It is a fact that the action potential curve recorded at a point distant from the stimulated point is composed of a few waves, and also that the conduction velocity of a fibre varies with its diameter, if measured separately. But it is quite another thing, whether the conduction velocity of a fibre of its own can be maintained even when it is excited with other quicker or slower fibres. There is a point here to be doubted. However, it is not a matter to be decided by speculation, and we ought to wait until the studies on interaction will be completed. Here we wish just to point to a new possibility of explaining the genesis of the composite action potential wave, by quoting the well known microscopic figure of a nerve section. We see there several groups of fibres, each'surrounded by a membrane called the perineurium. Each group is divided again into smaller groups, which are separated by finer membranes called the endoneurium. The rule for these groupings seems to be unknown yet, but whatever it may be, each group may have a conduction velocity of its own, provided that the separating membrane, supposedly the perineurium, is working as an insulating membrance as well.

\section{Summary}

Two or more iron core models of nerve were laid in parallel in a bath of strong nitric acid, and examinations were made on the interactions between them. The points obtained are as follows:

1) If parallel cores of nearly equal conduction velocities are stimulated simultaneously at one end, the activation waves are seen to be transmitted with one and the same velocity in every core. This "common" velocity is smaller than either of the individual ones, and it is the smaller, the larger the number of the cores is.

2) When two cores of different velocities are stimulated simultancously, they trans- 
mit their waves with a certain average velocity.

3) For the conduction velocity of a certain definite, core, the simple co-existence of other cores has no meaning.

4) In a core which is stimulated directly after the activation of a neighbouring core, , the wave is conducted with a larger velocity than when it is stimulated alone.

5) These phenomena were ascribed to the electric interaction between the cores.

6) Physiological meanings of these phenomena were discused and a new possibility was pointed out as to the genesis of the composite waves in the action potential curve obtained from a mixed nerve.

\section{REFERENCES}

1) Yamagiwa, K: This journal 1, 452, 1948.

2) Otani, T: Jap. J. of Med. Sciences III, Biophysics 4, 355, 1937.

3) Katz, B. and O. H. Schmitt: J. Physiol. 97, 471, 1940; 100, 369, 1942.

4) Blair, E. A. and J. Erlanger: Am. J. Physiol. 131, 483, 1936.

5) Renshaw, B. and P. O. Therman: Am. J. Physiol. 133, 96, 1941.

6) Marrazzi, A. S. and R. Lorente de Nó.: J. Neurophysiol. 7, 83, 1944.

The expences for this work were defrayed by a grant from the National Research Council of Japan. 